\title{
Análisis de la Transmisión de Tramas STM-N sobre un Enlace de Fibra Óptica DWDM
}

\author{
Julio C. Agudelo-Ramirez ${ }^{1}$ \\ Fabian G. Mejía-Builes ${ }^{2}$ \\ Nelson D. Gomez-Cardona ${ }^{3}$ \\ Claudia M. Serpa-Imbett ${ }^{4}$
}

\section{Resumen}

En este trabajo se presenta un análisis del desempeño de la capa física de un enlace DWDM de $66 \mathrm{~km}$ de fibra óptica monomodo sobre la cual se implementa un sistema SDH para envío de módulos STM-1 a 155 Mbps. Se muestran los resultados de la simulación computacional en Optisystem 7.0 (Versión Evaluación) para analizar las características de un sistema DWDM a una velocidad de 2.5 Gbps equivalente al envío de módulos STM-16. Los resultados de diagramas de ojo y BER muestran que es posible la distinción entre bits cuando el ruido térmico en el detector es inferior a $1 \times 10^{-19} \mathrm{~W} / \mathrm{Hz}$. Se analizan mediciones experimentales de Jitter y su correspondiente comparación con el valor normatizado por la ITU-T para módulos STM-1. Se propone el uso de más longitudes de onda para aprovechar al máximo la capacidad de transmisión de la fibra o la instalación de interfaces de mayor velocidad en los extremos del enlace para el incremento del ancho de banda disponible.

1 Facultad de Ingenieria, INSTITUTO TECNOLÓGICO METROPOLITANO, jagudelo@decom.com.co

2 Facultad de Ingenieria, INSTITUTO TECNOLÓGICO METROPOLITANO, fmejia@comcel.com.co

3 Centro de Investigación, INSTITUTO TECNOLÓGICO METROPOLITANO, nelsongomez@itm.edu.co

4 Centro de Investigación, INSTITUTO TECNOLÓGICO METROPOLITANO, claudiaserpa@itm.edu.co

Fecha de recepción: 13 de Agosto de 2010

Fecha de aceptación: 12 de Octubre de 2010 


\section{Palabras clave}

Efecto Brillouin, efectos no lineales, Jerarquía Digital Síncrona (SDH), Multiplexación por longitud de onda densa (DWDM), Synchronous Transport Module (STM).

\section{Abstract}

In this work, we present an analysis of physical layer performance of a DWDM-link of $66 \mathrm{~km}$ of single-mode optical fiber on which is implemented a SDH system to send STM-1 at 155 Mbps. We show the computational simulation in Optisystem 7.0 (Evaluation Version) to analyze the DWDM system characteristic at 2.5 Gbps equivalent to a STM-16 frame. The results of the eye diagram and BER show that is possible to distinguish between bits when the detector thermal-noise is less than $1 \times 10^{-19} \mathrm{~W} / \mathrm{Hz}$. We analyze experimental measurements of jitter and its corresponding comparison with the value normalized by the ITU-T for a STM-1 frame. The use of more wavelengths is proposed to maximize the transmission capacity of fiber optics or installation of interfaces with high capacity for increasing the available bandwidth.

\section{Keywords}

Brillouin effect, Dense Wavelenght Division Multiplexing (DWDM), non linear effects, Synchronous Digital Hierarchy (SDH), Synchronous Transport Module STM-N. 


\section{INTRODUCCIÓN}

Las redes multiplexadas por longitud de onda que permiten la inserción simultánea de múltiples longitudes de onda sobre un mismo hilo de fibra (WDM, Wavelenght Division Mulitplexing) han permitido una evolución flexible y rápida de las redes de fibra óptica actuales para dar solución a la demanda de requerimiento de mayor ancho de banda por parte de los usuarios finales (Dutta, 2004). Los sistemas WDM han sido utilizados en enlaces de fibra punto a punto, sin embargo hoy en día se propone su uso en la implementación de enlaces punto-multipunto para redes de acceso urbanas (Kazovski, 2007).

Existen diferentes tecnologías basadas en WDM y se clasifican de acuerdo a la separación espectral entre longitudes de onda consecutivas. Una de ellas, la DWDM (Dense WDM) está normatizada por la ITU-T G.692 que define la asignación de 43 longitudes de onda con separación de $0,4 \mathrm{~nm}$ entre longitudes de onda adyacentes (ITU-T, 2008). La DWDM es una tecnología concebida para ampliar la capacidad de las redes de fibra óptica, así como para permitir el crecimiento en volumen y complejidad de los servicios de telecomunicaciones demandados.

Uno de los estándares que normatiza la velocidad de información e interfaces de redes de fibra óptica punto-punto en redes troncales es el SDH (Synchronous Digital Hierarchical, o SONET para estándar Europeo) que envía señales a $155 \mathrm{Mbps}$, velocidad denominada el módulo de transporte síncrono de primer nivel o STM-1. A partir de STM-1 es posible la constitución de módulos de transporte STM-16 a 2.5 Gbps hasta STM-64 a 10 Gbps. Una red SDH tiene la desventaja de tener un ancho de banda insuficiente para las necesidades de transporte de servicios de video, voz y datos, por lo que se proponen sistemas SDHmultiplexados por longitud de onda SDH-DWDM para transporte de información con módulos STM-1 hasta STM-64 (Capmany \& Ortega, 2007).

El uso simultaneo de módulos STM-N (N=1, 4, 16, 64) sobre DWDM puede ocasionar el solapamiento entre canales adyacentes y cercanos, por lo que es necesario el análisis de registros espectrales para determinar la interferencia inter-canal y efectos 
no lineales por la alta potencia de las fuentes de luz (Mizoochi et al., 2003). Además, la velocidad de transmisión de módulos STM de mayor capacidad de transmisión trae como consecuencia degradaciones en la señal, debido a la dispersión cromática, la atenuación, y los efectos no lineales en la fibra que son observados como interferencia intersímbolo (ISI) y cuyo comportamiento se analiza con registros de BER (Bit error Rate ) y diagramas de ojo (Agrawal, 2007). De otra parte, en las redes SDH una de las causantes de errores en la transmisión está asociada a las variaciones temporales del instante en que se espera la llegada de bits al receptor (Al-Azzawi, 2007). Esta medida está relacionada con el valor del Jitter que consiste en un análisis de las desviaciones temporales de la señal muestreada. Los valores permitidos están normatizados por la ITU-T G.285 de 2000 para módulos STM-1 e indica los valores de las desviaciones temporales que no son causantes de errores en la recepción de la señal.

En este trabajo se presenta un análisis de un enlace puntopunto de $66 \mathrm{~km}$ de fibra óptica monomodo con una pérdida de 13,265 dB sobre el cual se implementa un sistema SDH-DWDM con envío de tramas STM-1. La arquitectura real del enlace y sus respectivos valores de pérdidas por conectorización son usados para realizar una simulación computacional en Optisystem 7.0 (Versión Evaluación) con el fin de analizar las características de un sistema DWDM implementado sobre este enlace a una velocidad de 2.5 Gbps, equivalente al envío de módulos STM-16.

Se muestran los resultados de diagramas de ojo y BER mostrando que es posible la distinción entre bits cuando el ruido térmico en el detector es inferior a 1x10-19 W/Hz. Finalmente se muestra una comparación del Jitter normatizado por la ITU-T con medidas experimentales sobre tramas STM-1. Se propone el uso de más longitudes de onda para aprovechar al máximo la capacidad de transmisión de la fibra o la instalación de interfaces de mayor velocidad en los extremos del enlace para el incremento del ancho de banda disponible. 


\section{MONTAJE EXPERIMENTAL}

El montaje experimental consiste en un enlace de $66 \mathrm{~km}$ para la transmisión de dos longitudes de onda simultáneamente usando un MUX de hasta 80 canales bajo el formato DWDM (separación entre longitudes de onda $\leq 0,8 \mathrm{~nm}$ ). El enlace está construido de fibra óptica monomodo (SMF, Single mode Fiber) con 24 empalmes por fusión con una atenuación por empalme de 0,026 hasta 0,086 dB. La pérdida total y la distribución de empalmes en la red fueron medidas con un OTDR FTB-7200 a $1550 \mathrm{~nm}$ y con un ancho de pulso de $500 \mathrm{~ns}$, dando como resultado una pérdida total en el enlace de 13,265 dB como se muestra en la Fig. 1.



Fig. 1. Montaje experimental SDH-WDM

\section{SIMULACIÓN COMPUTACIONAL}

La simulación computacional se realiza usando el paquete Optisystem V.7 (Versión Evaluación) para analizar el desempeño físico del enlace a una velocidad de transmisión de 2.5 Gbps por canal como se muestra en la Fig. 2. Los canales están constituidos por dos fuentes de luz láser que emiten a $1552,52 \mathrm{~nm}$ y 1551,72 $\mathrm{nm}$, con una separación de 0,8 $\mathrm{nm}$. Ambas fuentes de luz fueron conectadas a un modulador cuya entrada eléctrica consiste en un generador de secuencia de bits a 2.5 Gbps y un generador de 10 $\mathrm{GHz}$ en formado NRZ. Estas señales son la entrada del multiplexor óptico (MUX) que inserta ambas longitudes de onda sobre el enlace de fibra óptica descrito en el montaje experimental y cuyas características fueron usadas para la simulación. Al final de $\operatorname{los} 66 \mathrm{~km}$ de fibra rotulado como Sitio B, se coloca un demultiplexor óptico (DEMUX) de dos canales seguido por dos 
fotodetectores un filtro eléctrico pasabajo a $2 \mathrm{GHz}$. En Sitio A se coloca un Analizador de Espectros Ópticos (OSA, Optical Spectrum Analizer) y un medidor de potencia (PM, Power Meter) para analizar las características ópticas y espectrales de la señal de entrada al enlace, asimismo estos elementos se colocan al final del enlace a la salida del DEMUX. Adicionalmente, en Sitio B, se coloca un Analizador de BER para analizar la calidad de la señal de salida.



Fig. 2. Simulación Computacional en Optysistem V.7 (Versión evaluativa) del enlace de fibra óptica bajo una configuración SDH-WDM

\section{RESULTADOS}

\subsection{Mediciones Espectrales en el Enlace}

La Fig. 3a muestra la potencia óptica de cada longitud de onda a la salida del MUX. La potencia óptica máxima para cada longitud de onda: 1552,52 y 1551,72 nm es de aproximadamente 9,8 dBm para cada longitud de onda. La Fig. 3b muestra que la potencia óptica máxima de cada longitud de onda es de aproximadamente $2,7 \mathrm{dBm}$ medida a $33 \mathrm{~km}$, y se observa a $0,9 \mathrm{~nm}$ 
de las longitudes de onda de las señales principales la aparición equidistante de otras longitudes de onda con potencias de aproximadamente $-65 \mathrm{dBm}$ que muestra la aparición del efecto no lineal Brillouin debido a la alta potencia de las fuentes de luz portadoras (Agrawal, 2000). Asimismo, en la Fig. 3c se muestran la potencia óptica al final del DEMUX de aproximadamente -7,3 $\mathrm{dBm}$, como la presencia de las longitudes de onda que indican no linealidades en el enlace.

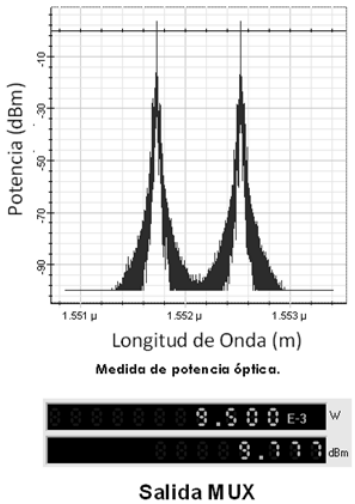

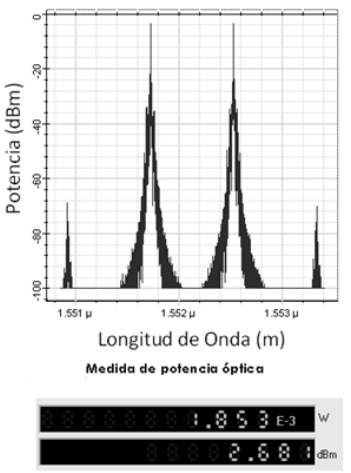

Mitad del Enlace

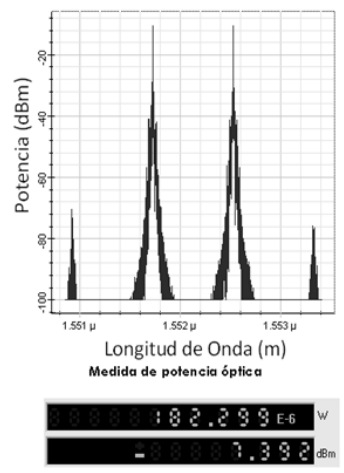

Entrada DeMux

Fig. 3 a) Registro espectral al inicio del enlace: MUX (sitio 1), b) Registro espectral a la mitad del enlace: $33 \mathrm{~km}$, c) Registro Espectral a la entrada del enlace: DEMUX (sitio 2)

\subsection{Análisis de Diagramas de Ojo y Medidas de BER a 2,5 Gbps}

Las medidas de diagrama de ojo se pueden observan en la Fig. 4. Se puede observar la variación del diagrama cuando el ruido térmico en el detector va desde $1 \times 10^{-20} \mathrm{~W} / \mathrm{Hz}$ hasta $1 \times 10-17 \mathrm{~W} / \mathrm{Hz}$. A medida que aumenta e ruido térmico se observa la degradación de la señal a $2.5 \mathrm{Gbps}$, y en los registros correspondientes a ruidos térmicos con valores de $1 \times 10-18 \mathrm{~W} / \mathrm{Hz}$ y $1 \times 10-17 \mathrm{~W} / \mathrm{Hz}$ no es posible distinguir entre un bit correspondiente a "uno" y uno correspondiente a "cero". 

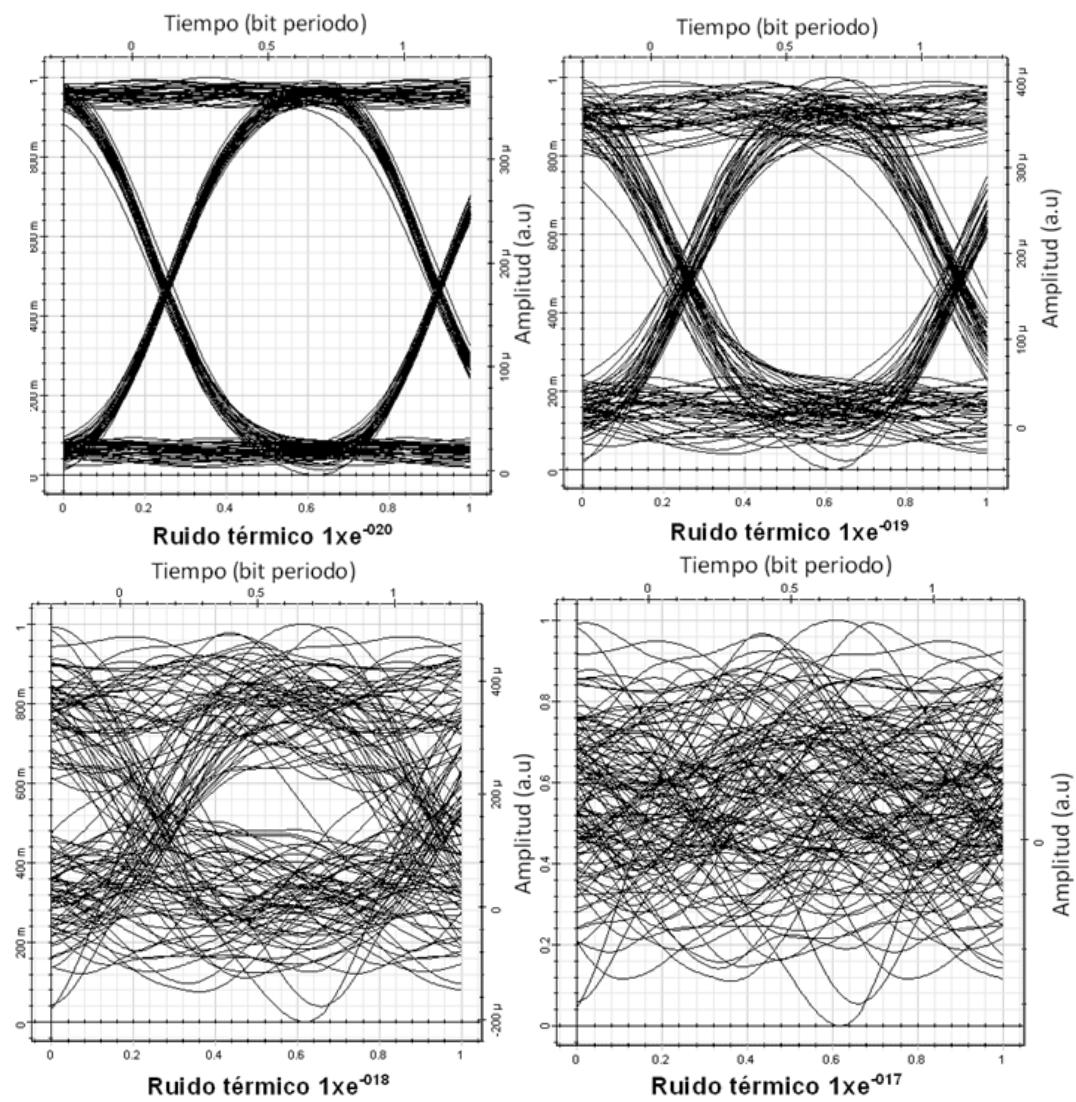

Fig. 4. Medida de diagramas de ojo para diferentes valores de ruido térmico medidos a la salida del DEMUX. Escala de tiempo 0,2 ns/div

El valor correspondiente a una transmisión libre de errores (error free) se tomará con ruidos térmicos inferiores a 1x10-19 $\mathrm{W} / \mathrm{Hz}$ valores para los cuales aún es posible la distinción de bits en la transmisión, y con un valor de BER mayor de 109 como lo indica la línea horizontal de la Fig. 5, donde se muestra la variación del BER vs. SNR o relación señal a ruido en escala semilogarítmica, y los valores para los cuales no es posible la distinción de bits en la transmisión que son los valores que están encima de la línea rotulada como "error free" (libre de errores). 




Fig. 5. Medida de BER vs. OSNR a la salida del DEMUX

\subsection{Jitter}

En la Fig. 6 muestra la medida experimental del Jitter en unidades de intervalo y su respectiva comparación con la curva recomendada por la norma G.285 de 2000 (máscara de tolerancia del Jitter). Los valores por encima de la máscara de tolerancia del Jitter indican un adecuado funcionamiento en la sincronización de la recepción de la información en el receptor optoelectrónico. El Eje horizontal muestra la razón de muestreo de la señal en unidades de $\mathrm{kHz}$, el eje vertical muestra la amplitud pico a pico en unidades de Intervalo del bit enviado que va de 1 bps a $10 \mathrm{kbps}$ para el caso de la medición en el receptor o DEMUX como se muestra en la Fig. 6 a y de 1 bps - 1 kbps para el caso de la medición en el transmisor o DEMUX como e muestra en la Fig. 6 b. El símbolo " $\Delta$ "es el valor más bajo de las fluctuaciones de frecuencia admisible, y el símbolo “+” es el valor máximo tolerado para la fluctuación de frecuencia. 

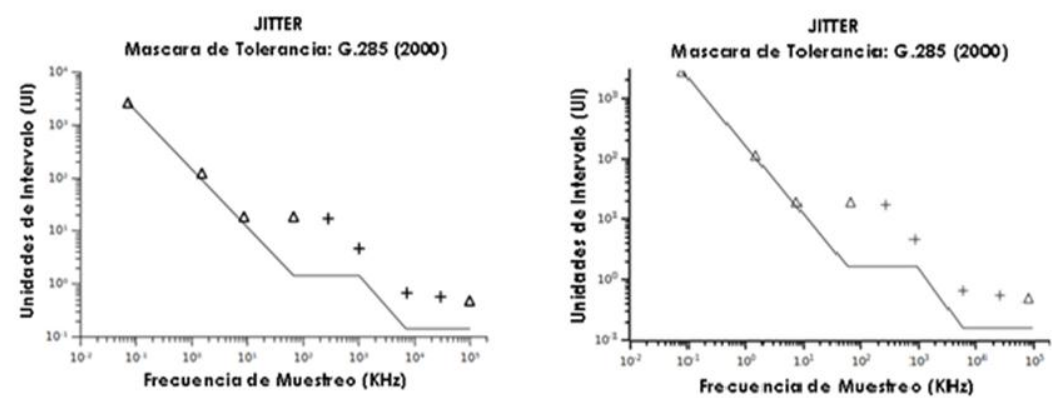

Fig. 6. Medidas de JITTER para el enlace de $66 \mathrm{~km}$, a) En el DEMUX, b) en el MUX

\section{DISCUSIÓN}

En la Fig. 4 se muestran el comportamiento y degradación de la señal a 2.5 Gbps a medida que aumenta el ruido térmico en el detector. La curva de BER muestra que en el enlace de $66 \mathrm{~km}$ de fibra es posible la transmisión de señales a $2.5 \mathrm{Gbps}$ libre de errores, cuando el ruido térmico es menor de $1 \times 10-19 \mathrm{~W} / \mathrm{Hz}$, Se propone un incremento en la velocidad de la señal del estándar SDH a con tramas STM-1 a 155 Mbps hasta 2.5 Gbps con tramas STM-64 sin problema por degradación de la señal por atenuación, efectos dispersivos y no lineales (Sivalingam, 2005) y con ruidos menores a 1x10-19 W/Hz. Para la implementación de tramas STM64 es necesario la medición de Jitter a 2.5 Gbps y su comparación con los valores permitidos para garantizar la integridad de la señal cuando hay aumento de la velocidad (de $155 \mathrm{Mbps}$ hasta 2.5 Gbps), ya que se espera un incremento de los errores debido al muestreo y sincronización de la señal en el receptor. Las medidas de Jitter muestran el adecuado funcionamiento del sistema bajo el estándar SDH con tramas STM-1 a 155 Mbps.

\section{CONCLUSIONES}

El enlace mostrado es utilizado para el transporte de información bajo una configuración DWDM-SDH con una 
capacidad por canal de 155 Mbps. Este sistema soporta por canal hasta 19 llamadas de voz en curso a $8 \mathrm{Mbps}$. Se propone el uso de más longitudes de onda para aprovechar al máximo la capacidad de transmisión de la fibra óptica y la capacidad de operación de MUX implementado en el enlace que dispone de hasta 80 longitudes de onda. Basados en los resultados de BER y diagramas de ojo a $2.5 \mathrm{Gbps}$, se propone la instalación de interfaces de mayor velocidad en los extremos del enlace, teniendo en cuenta las medidas de jitter para redes óptica recomendados por la ITU-T, que garantice el adecuado desempeño de la red a velocidades mayores, o el incremento y uso de más longitudes de onda en el sistema DWDM ya existente (Sivalingan, 2000)

\section{AGRADECIMIENTOS}

Este trabajo se realizó con información suministrada por una Empresa Proveedora de Servicios basada en redes SDH. El nombre de la empresa hace parte de la reserva de información de la cooperación investigativa. Este reporte hace parte de los resultados del proyecto Código: P07203 financiado por el INSTITUTO TECNOLÓGICO METROPOLITANO Institución Universitaria de Medellín-Colombia. Se le agradece a Optisystem por el acceso a la versión de evaluación del programa de simulación.

\section{REFERENCIAS}

Agrawal, G., (2007); Fiber-optic communication systems. Michigan: WileyInterscience.

Al-Azzawi, A., (2007); Fiber optics: principles and practices: Optical Science And Engineering Series. Boca Raton: Optical Science And Engineering Series.

Capmany, J., Ortega, B., (2007); Redes Opticas. Valencia: Unviersidad Politecnica de Valencia.

Dutta, N.K., (2004); WDM technologies: Optical Network. New York: Academic Press. 
ITU-T, (2008); www.itu.int. Recuperado el 7 de 03 de 2009, de International Union Telecommunication: www.itu.int

Kazovsky, L.G., Shaw, W., Gutierrez, D., Cheng, N., (2007); NextGeneration on Optical Access Network. Journal of Lightwave Technology, 3428-3442.

Mizuochi, T.; Ishida, K.; Kobayashi, T.; Abe, J.; Kinjo, K.; Motoshima, K.; Kasahara, K., (2003); A comparative study of DPSK and OOK WDM transmission over transoceanic distances and their performance degradations due to nonlinear phase noise. Journal of Lightwave Technology, 1933-1943

Sivalingam, K., (2005); Emerging optical network technologies: architectures, protocols, and performance. Washington: Springer.

Sivalingam, K., (2000); Optical WDM networks: principles and practice. New York: Springer. 